\title{
BMJ Open Exploring the added value of hospital- registry data for showing local service outcomes: cancers of the ovary, fallopian tube and peritoneum
}

$\overline{\text { David Roder, }{ }^{1} \text { Margaret Davy, }{ }^{2} \text { Sid Selva-Nayagam, }{ }^{3} \text { Sellvakumaran Paramasivam, }{ }^{4}}$ Jacqui Adams, ${ }^{5}$ Dorothy Keefe, ${ }^{6}$ Caroline Miller, ${ }^{7,8}$ Kate Powell, ${ }^{7,9}$ Kellie Fusco, ${ }^{1}$ Dianne Buranyi-Trevarton, ${ }^{9}$ Martin $\mathrm{K}$ Oehler $^{10}$

To cite: Roder D, Davy M, Selva-Nayagam S, et al. Exploring the added value of hospital-registry data for showing local service outcomes: cancers of the ovary, fallopian tube and peritoneum. BMJ Open 2019;9:e024036. doi:10.1136/ bmjopen-2018-024036

- Prepublication history for this paper is available online. To view these files, please visit the journal online (http://dx.doi org/10.1136/bmjopen-2018024036).

Received 7 May 2018 Revised 23 October 2018 Accepted 18 December 2018

Check for updates

(C) Author(s) (or their employer(s)) 2019. Re-use permitted under CC BY-NC. No commercial re-use. See rights and permissions. Published by BMJ.

For numbered affiliations see end of article.

Correspondence to Professor David Roder; David.Roder@unisa.edu.au

\section{ABSTRACT}

Objectives To explore the added value of hospital-registry data on invasive epithelial ovarian, tubal and peritoneal cancers.

Design Historic cohort analyses.

Methods Unadjusted and adjusted regression.

Setting Major South Australian hospitals.

Participants 1596 women (1984-2015 diagnoses).

Results 5 -Year and 10 -year survival was $48 \%$ and

$41 \%$, respectively, equivalent to relative survival for

Australia and the USA. After adjusting for age, clinical and geographic factors, risk of ovarian cancer death was $25 \%$ lower in 2010-2015 than 1984-1989. Women generally had surgical treatment $(87 \%)$ in their first round of care. This was more common for younger patients (adjusted OR ( $95 \%$ Cls) 0.17 (0.04 to 0.65 ) for $80+$ vs $<40$ years) and earlier International Federation of Gynecology and Obstetrics stages (adjusted OR 0.48 (0.13 to 1.78) for stage IIIB/C and 0.13 (0.04 to 0.45$)$ for stage IV vs stage IA). Most (74\%) had systemic therapy, which was more common for advanced stages (adjusted ORs $>15.0$ for stages III and IV vs stage IA). Few (9\%) had radiotherapy. Women generally had systemic therapy $(74 \%)$, without difference by service accessibility and socioeconomic disadvantage, suggesting equity. However, surgery was less common for residents of the most compared with least remote areas (adjusted OR 0.49 (0.24 to 0.99)); and more common prior to adjustment in the highest versus lowest socioeconomic category (unadjusted OR 1.55 (1.01 to 2.39)), but this elevation did not apply after adjustment (adjusted OR 0.19 (0.63 to 2.25)), with the difference largely explained by stage.

Conclusions Hospital-registry data add value for assessing local service delivery. Equivalent survival to Australia-wide and USA survival, and temporal gains after adjusting for stage and other patient characteristics are reassuring. Survival gains may reflect therapeutic benefits of more extensive surgery and improved chemotherapy regimens.

\section{INTRODUCTION}

The age-adjusted incidence of ovarian cancer in Australia is about 25\% higher than
Strengths and limitations of this study

- The study demonstrates the added value of hospital registries in providing: key prognostic and treatment data not available in Australian population-based registries and local data for local hospital service monitoring and evaluation.

- The usefulness of this hospital-registry model for more remote and smaller hospital settings is untested.

- In view of the more limited specialist support available for care in many of these settings, additional testing is indicated for these hospitals.

estimated worldwide, similar to rates for Western Europe, and lower than for other populations classified in GLOBOCAN as 'more developed' (eg, the UK and North America). ${ }^{1}$ The pattern of ovarian cancer mortality is similar, with the Australian rate exceeding the world estimate by $19 \%$, but lower than for other 'more developed' countries. ${ }^{1}$ About 1580 Australian women are diagnosed with ovarian cancer and around 1050 women die from it each year. ${ }^{23}$

Australian data show a reduction in age-standardised ovarian cancer incidence of approximately $14 \%$ since 1982 when national incidence data were first collected, and a mortality reduction of $19 \% \cdot{ }^{23}$ Mortality reductions have also been reported during the last 20 years in North America, Europe, South America and Japan. ${ }^{4}$ By comparison, trends in recorded incidence have been inconsistent, potentially due to differences in imaging practices, other diagnostic technology and changes in the use of oral contraceptives and menopausal hormones. ${ }^{5}$ Clearly, there are many factors since 1982 that could have affected incidence and mortality trends, among them, prevalence of obesity, changes 
in surgery and chemotherapy, use of hormone replacement therapy, tobacco smoking, reproductive history and use of contraceptive pills. ${ }^{4-6}$

In Australia, 5-year survival increased from $34 \%$ in 1984-1988 to $44 \%$ in $2009-2013 .{ }^{36}$ Similar trends have been reported by the USA Surveillance, Epidemiology and End Results Programme (SEER), indicating a 47\% 5-year survival for 2007-2013. ${ }^{7}$ Survival increases have been attributed to a greater emphasis on debulking and use of adjuvant platin and taxane-based therapies. ${ }^{8}$ Predictably stage at diagnosis is a key determinant of survival, with SEER data indicating a 5-year relative survival of $93 \%$ for localised disease, $73 \%$ for regional spread and $29 \%$ for women with metastatic disease. ${ }^{7}$

Although treatment may have contributed to increased survival in Australia, this cannot be verified with population-wide data which lack information on stage at diagnosis, other prognostic indicators and treatment. ${ }^{6}$ Treatment and risk-adjusted survival data are also lacking for most local clinical settings, which reduces capacity for local evaluation.

This study uses hospital-registry data from major hospitals in South Australia that include stage, grade and treatment. A previous benchmark study at these hospitals in 1984-1998 showed that approximately $90 \%$ of ovarian cancers had surgery in their primary course of care and $71 \%$ had systemic therapies. Combination surgery and adjuvant systemic therapies were provided to $66 \%$, whereas $6 \%$ had systemic therapy only. Only 5\% had radiotherapy. ${ }^{9}$ Updated data are presented now for these same hospitals covering diagnostic years up to 2015 and trends are explored by sociodemographic and tumour characteristics.

Our expectation, based on USA SEER data, is that stage-adjusted survivals would have increased during 1984-2015 and that survival for all stages combined would be similar to survival for Australia overall and the USA. $^{3}{ }^{67}$ We expect surgery and systemic therapies to remain the most common treatment choice, particularly for younger women, and for those diagnosed with local and regional disease, with a trend towards more extensive surgery and increased use of combined platin and taxanebased therapies. ${ }^{8}$

The principal objective in this study is to assess the added value of hospital-registry data as a complement to population-based data for local hospital review. ${ }^{9}$ With these hospital-registry data, clinicians can review disparities in treatment uptake across their patient groups and consider possible causes and solutions.

\section{METHODS}

The South Australian hospital-registry network was established in the 1980s, covering four major metropolitan hospitals that treated $60 \%$ of ovarian cancers diagnosed in South Australia during the present study period. ${ }^{9}$ These registries are authorised under Section 64, Part 7 of the South Australian Health Care Act (2008) to collect data on the quality and outcomes of care..$^{9} 10$ The four hospitals included South Australian major teaching hospitals. All used multidisciplinary teams located at these major public hospitals for treatment planning and evaluation.

This study included the 1596 invasive epithelial cancers of the ovary, fallopian tube and peritoneum diagnosed at study hospitals in 1984-2015. Patients were classified by: age at diagnosis; postcode-derived Socio-Economic Indexes for Areas index of relative socioeconomic disadvantage; geographic remoteness ('low' referring to major city areas and the remaining country areas divided arbitrarily into moderately remote and highly remote by Accessibility/Remoteness Index scores) and tumour characteristics (table 1). ${ }^{9} 1112$ The Strengthening the Reporting of Observational Studies in Epidemiology cohort reporting guidelines were used in this study. ${ }^{13}$

Anatomic primary site was coded using C56/57.0/48.1/48.2 codes of the International Classification of Diseases for Oncology, V.3 (ICD-O-3). Epithelial cancer data were extracted using ICD-O-3 codes for serous, mucinous, endometrioid, clear cell, other adenocarcinomas (not otherwise specified) and unknown epithelial lesions.

First-round treatment was defined as treatment following diagnosis, generally within $6-12$ months. Treatment was classified as surgery (except when for diagnostic purposes only)—specified as unilateral salpingo-oophorectomy, bilateral salpingo-oophorectomy, hysterectomy, omentectomy, debulking and exenteration and according to whether systemic therapy and radiotherapy were provided.

Death data were extracted from official death files, and for deaths occurring outside of South Australia, from the National Death Index. ${ }^{9}$ Causes-of-death were corrected when clinical data indicated this to be appropriate. ${ }^{9}$ The extent of loss to follow-up of deaths has been checked previously through active tracing and comparison with external case series, and found to be minimal, with little effect on calculated survival. ${ }^{9} 1214$

Disease-specific survivals were derived in historic cohort analyses using Kaplan-Meier product-limit estimates, with censoring of live patients on 31 December $2015 .{ }^{1516}$ This method was preferred to relative survival because risks of deaths from competing causes could not be assumed to be equivalent to population norms (an underlying assumption for relative survival) due to referral of highrisk patients (including those with extensive comorbidity) to these major hospitals. ${ }^{9}$

Population-based data show disease-specific survival, based on South Australian registry coding, to be a good proxy for relative survival, for example, a 1977-2003 study gave survival estimates for ovarian cancer of $36 \%$ at 5 years and $31 \%$ at 10 years, both for relative and disease-specific survival. ${ }^{17}$ This validation is important because cause-specific survival has been shown in other settings to be affected by variations in cause-of-death coding. ${ }^{18}$

Cox proportional hazards regression was used to analyse differences in disease-specific survival by demographic 
Table 1 Per cent case survival from cancer of the ovary, fallopian tube and peritoneum by period postdiagnosis; South Australian major public hospitals, 1984-2015 diagnoses*

\begin{tabular}{|c|c|c|c|c|c|c|c|}
\hline Number of cases & $\begin{array}{l}\text { 1-year } \\
\text { survival }\end{array}$ & $\begin{array}{l}\text { 2-year } \\
\text { survival }\end{array}$ & $\begin{array}{l}5 \text {-year } \\
\text { survival }\end{array}$ & $\begin{array}{l}10 \text {-year } \\
\text { survival }\end{array}$ & $\begin{array}{l}\text { 20-year } \\
\text { survival }\end{array}$ & $\mathrm{P}$ value† & $\begin{array}{l}\text { Adjusted HR } \\
\text { (95\% Cls) }\end{array}$ \\
\hline All $(n=1596)$ & 81.7 & 66.8 & 47.7 & 41.2 & 38.9 & - & - \\
\hline$<40(n=103)$ & 90.3 & 84.3 & 79.2 & 72.2 & 64.4 & \multirow[t]{4}{*}{$<0.001$} & 1.00 \\
\hline $40-49(n=210)$ & 92.8 & 82.8 & 65.8 & 56.3 & 53.9 & & $1.13(0.75$ to 1.71$)$ \\
\hline $70-79(n=324)$ & 71.7 & 55.2 & 34.7 & 29.6 & 29.6 & & 1.87 (1.28 to 2.73$)$ \\
\hline $80+(n=167)$ & 64.3 & 54.2 & 43.5 & 42.0 & - & & 1.63 (1.08 to 2.46$)$ \\
\hline \multicolumn{8}{|l|}{ FIGO stage: } \\
\hline IA $(n=212)$ & 99.5 & 96.1 & 89.0 & 86.1 & 80.8 & \multirow[t]{5}{*}{$<0.001$} & 1.00 \\
\hline IIIA $(n=320)$ & 79.9 & 60.1 & 36.6 & 29.6 & 27.4 & & 5.16 (3.54 to 7.53$)$ \\
\hline IIIB/C (n=376) & 82.9 & 64.8 & 35.4 & 25.5 & 23.9 & & 5.43 (3.71 to 7.96$)$ \\
\hline IV $(n=320)$ & 62.8 & 42.3 & 23.2 & 20.6 & 20.1 & & 7.38 (5.06 to 10.77$)$ \\
\hline Unknown $(n=91)$ & 64.5 & 47.7 & 37.3 & 34.7 & 34.7 & & 5.68 (3.59 to 8.98$)$ \\
\hline \multicolumn{8}{|l|}{ Differentiation: } \\
\hline Well $(n=148)$ & 95.2 & 90.2 & 82.1 & 78.9 & 76.8 & \multirow[t]{4}{*}{$<0.001$} & 1.00 \\
\hline Moderate $(n=311)$ & 85.8 & 71.1 & 57.5 & 50.9 & 47.6 & & 2.00 (1.35 to 2.97$)$ \\
\hline Poorly/undifferentiated $(n=841)$ & 79.4 & 63.8 & 39.7 & 31.4 & 29.9 & & 2.33 (1.60 to 3.40$)$ \\
\hline Unknown (n=296) & 77.4 & 58.9 & 42.5 & 39.6 & 35.6 & & 2.23 (1.50 to 3.32$)$ \\
\hline \multicolumn{8}{|l|}{ Histology: } \\
\hline \multicolumn{8}{|l|}{ Socioeconomic (SEIFA): } \\
\hline Low $(n=495)$ & 81.5 & 65.8 & 44.9 & 38.9 & 35.9 & \multirow[t]{4}{*}{0.364} & 1.00 \\
\hline Low/med $(n=398)$ & 79.2 & 64.3 & 48.0 & 41.8 & 39.6 & & $0.96(0.80$ to 1.15$)$ \\
\hline Med/high $(n=315)$ & 81.7 & 67.2 & 49.7 & 44.3 & 42.2 & & 0.93 (0.77 to 1.13$)$ \\
\hline High $(n=388)$ & 84.6 & 70.3 & 49.2 & 41.2 & 39.5 & & 0.91 (0.76 to 1.08$)$ \\
\hline \multicolumn{8}{|l|}{ Geographic remoteness: } \\
\hline Low $(n=1326)$ & 81.4 & 66.1 & 46.8 & 40.3 & 37.8 & \multirow[t]{3}{*}{0.176} & 1.00 \\
\hline Moderate $(n=128)$ & 81.2 & 67.2 & 51.8 & 44.4 & 43.4 & & 0.80 (0.62 to 1.04$)$ \\
\hline High $(n=142)$ & 85.4 & 73.3 & 52.7 & 46.6 & 45.2 & & 0.84 (0.65 to 1.08$)$ \\
\hline \multicolumn{8}{|l|}{ Diagnostic period (calendar years) } \\
\hline $1984-1989(n=285)$ & 75.5 & 58.5 & 49.7 & 42.9 & 40.1 & \multirow[t]{4}{*}{0.687} & 1.00 \\
\hline $1990-1999(n=596)$ & 80.2 & 67.0 & 47.0 & 42.3 & 40.6 & & 0.87 (0.72 to 1.05$)$ \\
\hline $2000-2009(n=461)$ & 85.5 & 68.1 & 47.1 & 38.0 & - & & 0.87 (0.71 to 1.06$)$ \\
\hline $2010-2015(n=254)$ & 85.7 & 74.6 & 44.1 & - & - & & 0.75 (0.57 to 0.98$)$ \\
\hline
\end{tabular}

*Kaplan-Meier product-limit disease-specific estimates; date of censoring of live cases-31 December 2015.

†Derived from unadjusted Cox proportional hazards regression.

$\ddagger$ Derived from Cox proportional hazards regression, adjusting for other variables in the table.

FIGO, International Federation of Gynecology and Obstetrics; NOS, not otherwise specified; SEIFA, Socio-Economic Indexes for Areas. 
and clinical characteristics. This was undertaken using the same follow-up period and censoring rules as for the Kaplan-Meier analyses. ${ }^{15} 16$ Assumptions underlying multivariable Cox regression analyses, including proportionality (tested using Schonfield's residuals) and lack of co-linearity, were met. Results were very similar when missing values were filled using multiple imputation, when complete case analysis was substituted and when the Cox analyses were replaced by competing risk regression. $^{1516}$

First-round treatment was analysed by person and tumour characteristic using the Pearson's $\chi^{2}$ statistic or Mann-Whitney $\mathrm{U}$ test, depending on whether variables were binary, multinominal or distributed on an ordinal scale. ${ }^{15} 16$ In addition, unadjusted and multivariable logistic regression were used. ${ }^{1516}$

Multivariable analyses were also undertaken to assess whether hospital site was predictive of outcomes and whether there was evidence of clustering and effect modification by hospital. ${ }^{16}$ For example, when individual hospital sites were included in the proportional hazards regression model as dummy variables, they were not significant predictors $(\mathrm{p}>0.400)$, nor did adding them affect the coefficients for other predictors. This was not unexpected. These public hospitals are part of the one South Australian Cancer Service, which provides a central coordinating role. All have access to common clinical guidelines and standards, and clinicians frequently moving between them. Also, clustering by hospital was not suggested when employing multilevel mixed-effect modelling by regarding the data as nested by hospital site, and by testing the clustering effect by hospital using the variable for hospital as the random effect.

\section{Patient and public involvement}

The development of the registry and its workplans has had substantial patient and consumer involvement through a formalised planning and monitoring process.

Ongoing workplans and operations are monitored and reviewed by funders, including the Cancer Council South Australia, which contributes through the Beat Cancer Project. Specialist clinics identify topics for review, of which some are based on/prompted by the questions raised by their patients.

The use of the registry for this study was approved by the Department of Health Research Ethics Committee and University of South Australia, both with active consumer involvement, thus providing another layer of public and consumer input.

This study involved the use of routinely collected registry data specifically authorised under state law and planned by clinical experts and consumers.

Participants all attended specialised gynaecological oncology clinics with whom we work. We work with these clinics in developing consumer messages for distribution to their patients and other relevant stakeholder groups.
RESULTS

\section{Stage}

International Federation of Gynecology and Obstetrics (FIGO) stage was recorded for $94 \%$ of patients and distributed as follows: IA-14\%; IB/C-9\%; IIA-2\%; IIB/C-8\%; IIIA-21\%; IIIB/C-25\% and IV-21\%. B and $\mathrm{C}$ subcategories were combined due to less specific coding in the earlier years. Stage distributions did not differ by diagnostic period (Spearman's $\mathrm{p}=0.660$ ).

\section{Survival}

Survival was $48 \%$ at 5 years and $41 \%$ at 10 years during 1984-2015 (table 1). Survival percentages reduced with: (1) increasing stage $(p<0.001)$; (2) higher tumour grade $(\mathrm{p}<0.001)$ and $(3)$ older age at diagnosis $(\mathrm{p}<0.001)$. There was the indication of higher survival for women aged $80+$ than 70-79 years, but this was not statistically significant $(\mathrm{p}=0.236)$.

Survival percentages varied by histology type $(\mathrm{p}<0.001)$-higher 5 -year survival for endometrioid $(78 \%)$, clear cell $(68 \%)$ and mucinous $(65 \%)$ adenocarcinomas than for adenocarcinomas not otherwise specified $(38 \%)$ and serous adenocarcinomas $(42 \%)$. Survival did not vary by diagnostic period $(\mathrm{p}=0.687)$, geographic remoteness $(\mathrm{p}=0.176)$ or socioeconomic disadvantage $(\mathrm{p}=0.364)$.

Multivariable analysis confirmed a lower survival with: (1) increasing stage-HR (95\% CIs) increasing to 7.38 (5.06 to 10.77) for stage IV compared with stage IA; (2) higher grade-HR increasing to 2.33 (95\% CIs 1.60 to $3.40)$ for poorly or undifferentiated compared with well-differentiated lesions and (3) older age at diagnosis-HR increasing to 1.87 (95\% CIs 1.28 to 2.73 ) for $70-79$ years and 1.63 (95\% CIs 1.08 to 2.46 ) for $80+$ years women compared with $<40$ years (table 1 ).

Endometrioid adenocarcinomas had the lowest adjusted HR of 0.62 (95\% CIs 0.45 to 0.86 ) compared with serous adenocarcinomas as the reference. By comparison, non-specified adenocarcinomas had a higher adjusted HR at 1.20 (95\% CIs 1.03 to 1.40). Notably, a downward gradient in HR was evident to a low of 0.75 (95\% CIs 0.57 to 0.98 ) for 2010-2015 compared with the 1984-1989 diagnostic period.

The geographic measures of remoteness and socioeconomic status did not show survival gradients (table 1).

\section{Any treatment}

Overall, $95 \%$ of patients were recorded as receiving some treatment for their cancer (ie, surgery, radiotherapy and/ or systemic therapy). The percentage decreased with: age $(p<0.001)$ and increase in stage $(p<0.001)$. Differences were evident by histology type $(p<0.001)$, with the treated percentage varying from $99 \%$ for endometrioid and clear cell adenocarcinomas to $89 \%$ for non-specified adenocarcinomas. Differences were also observed by tumour grade $(p<0.001$, with the treated percentage reducing with higher grade. By comparisons, differences were not observed by socioeconomic status $(\mathrm{p}=0.113)$, 
remoteness of residential area $(\mathrm{p}=0.598)$ or diagnostic period $(\mathrm{p}=0.693)$.

Adjusted analysis, including all variables in table 1 as predictors of treatment, confirmed a decrease in OR of being treated with increasing age, advanced stage and non-specified compared with serous adenocarcinomas, but these differences were not statistically significant ( $p>0.200)$. Differences were not found by tumour grade, socioeconomic status, geographic remoteness or diagnostic period.

\section{Surgery}

Approximately $87 \%$ of patients had surgical treatment (table 2). This percentage reduced with: (1) increasing stage $(p<0.001)$; $(2)$ higher tumour grade $(p=0.021)$ and (3) older age at diagnosis $(\mathrm{p}<0.001)$. A difference was also found by histology type $(p=0.002)$, with the highest surgical coverage relating to endometrioid $(99 \%)$ and clear cell $(95 \%)$ adenocarcinomas, followed by serous $(92 \%)$, mucinous $(90 \%)$ and non-specified adenocarcinomas $(69 \%)$. The percentage having surgical treatment did not vary by diagnostic period ( $\mathrm{p}=0.986)$, geographic remoteness $(\mathrm{p}=0.507)$ or socioeconomic status (although approaching statistical significance $(\mathrm{p}=0.058)$ ), with an unadjusted OR of 1.55 (95\% CI 1.01 to 2.39 ) for the highest versus lowest socioeconomic category.

A trend towards more extensive surgery was evident. In the 1990 s, about $37 \%$ had debulking or exenteration, whereas this proportion increased to approximately $71 \%$ by $2010-2015$. There was also a trend towards greater use of adjunctive systemic therapy for surgical cases, with the percentage having this therapy increasing from $64 \%$ in the 1990 s to $73 \%$ in $2000-2015$. Of surgical cases having systemic therapies, about $9 \%$ were neoadjuvant and $91 \%$ were adjuvant in the 1990s, compared with $17 \%$ and $83 \%$, respectively for 2000-2015.

Multivariable analysis confirmed the lower OR for surgery with: (1) increasing stage and (2) older age at diagnosis (table 2). The OR for surgery was confirmed to be low for non-specified compared with serous adenocarcinomas as the reference at 0.28 (95\% CI 0.17 to 0.45$)$.

Geographic measures of residential remoteness showed a reducing OR for surgery with increasing remoteness, reaching 0.49 (95\% CI 0.24 to 0.99 ) for the most compared with least remote category. By comparison, a difference was not seen by level of socioeconomic disadvantage (table 2), where the unadjusted higher OR for high versus low socioeconomic status was largely explained by stage.

\section{Radiotherapy}

Radiotherapy was rarely used for these cancers, applying to only $9 \%$ of patients for $1984-2015$ and $4 \%$ for $2010-$ 2015 (table 3). There was a reduction in use of radiotherapy with increasing age $(\mathrm{p}<0.001)$. Differences were not observed by stage $(\mathrm{p}=0.696)$, histology type $(\mathrm{p}=0.865)$, grade $(\mathrm{p}=0.783)$, diagnostic period $(\mathrm{p}=0.119)$, geographic remoteness $(\mathrm{p}=0.372)$ or socioeconomic disadvantage $(\mathrm{p}=0.716)$.

Multivariable analysis confirmed a reduction in OR for radiotherapy with increasing age (OR of $0.05(95 \%$ CI 0.01 to 0.41 ) for $80+$ years compared with $<40$ years) (table 3). A difference was suggested by stage, with higher OR of 2.62 (95\% CI 1.05 to 6.52 ) for stage IB/C and 3.48 (95\% CI 1.47 to 8.26 ) for stage IIB/C compared with stage 1A. Differences were not observed by histology type, grade or geographic measures of remoteness or socioeconomic disadvantage (table 3 ). There was a difference by diagnostic year with a low OR for radiotherapy of 0.36 (95\% CI 0.14 to 0.92) for 2010-2015 compared with 1984-1989.

\section{Systemic therapy}

Overall, $74 \%$ of patients received systemic therapy, with the percentage varying by age $(\mathrm{p}<0.001)$-from $82 \%$ for 50-69 years to $40 \%$ for $80+$ years (table 4 ). Generally, systemic therapy was provided with surgery, although $8 \%$ had systemic therapy without other accompanying treatment (increasing from $7.7 \%$ in $1984-1989$ to $9.1 \%$ in 2010-2015).

Differences were evident in percentages exposed to systemic therapy by: histology type $(\mathrm{p}<0.001)$ - a comparatively high proportion had systemic therapy for serous adenocarcinomas $(84 \%)$ and a low proportion for mucinous lesions $(50 \%)$; stage $(\mathrm{p}<0.001)$ - a relatively low proportion of stage IA cases had this treatment $(33 \%)$ compared with stages IB/C-IV (68\%-87\%) and grade $(\mathrm{p}<0.001)$-an increasing proportion of patients with lower levels of differentiation having systemic therapy. An increase was suggested between 1984-1999 and 20002015 diagnostic periods $(\mathrm{p}=0.003)$, although a lower percentage was suggested for 2010-2015 than 2000-2009 (table 4). Differences were not observed by geographic remoteness $(\mathrm{p}=0.600)$ or socioeconomic disadvantage $(\mathrm{p}=0.750)$.

Of patients treated with systemic therapies in 19841998, 65\% had a platin-based therapy, $64 \%$ had cyclophosphamide and $5 \%$ had doxorubicin. Systemic therapy types changed over time towards a greater use of carboplatin and paclitaxel; by 2010-2015, around $91 \%$ of those treated with systemic therapies had carboplatin and $67 \%$ had both carboplatin and paclitaxel. Conversely, use of cyclophosphamide was no longer a treatment of choice.

Adjusted analyses confirmed the low OR of systemic therapy for patients aged $80+$ years at 0.07 (95\% CI 0.03 to 0.15$)$ compared with $<40$ years as the reference. Compared with serous adenocarcinomas, the OR for this therapy was low for mucinous lesions at 0.40 (95\% CI 0.23 to 0.69$)$ and non-specified adenocarcinomas at $0.63(95 \%$ CI 0.42 to 0.95 ). The higher the stage the greater were the OR for systemic therapy up to stage IIIB/C compared with stage IA (21.42 (95\% CI 11.77 to 38.98)). A high OR also applied to stage IV at 15.68 (95\% CI 8.80 to 27.92). Compared with 1984-1989, an elevated OR applied to the 2000-2015 diagnostic period of 1.95 (95\% CI 1.24 to 
Table 2 Per cent cancers of the ovary, fallopian tube and peritoneum treated by surgery (and ORs of surgery) as part of the primary course of treatment; South Australian major public hospitals, 1984-2015 diagnoses

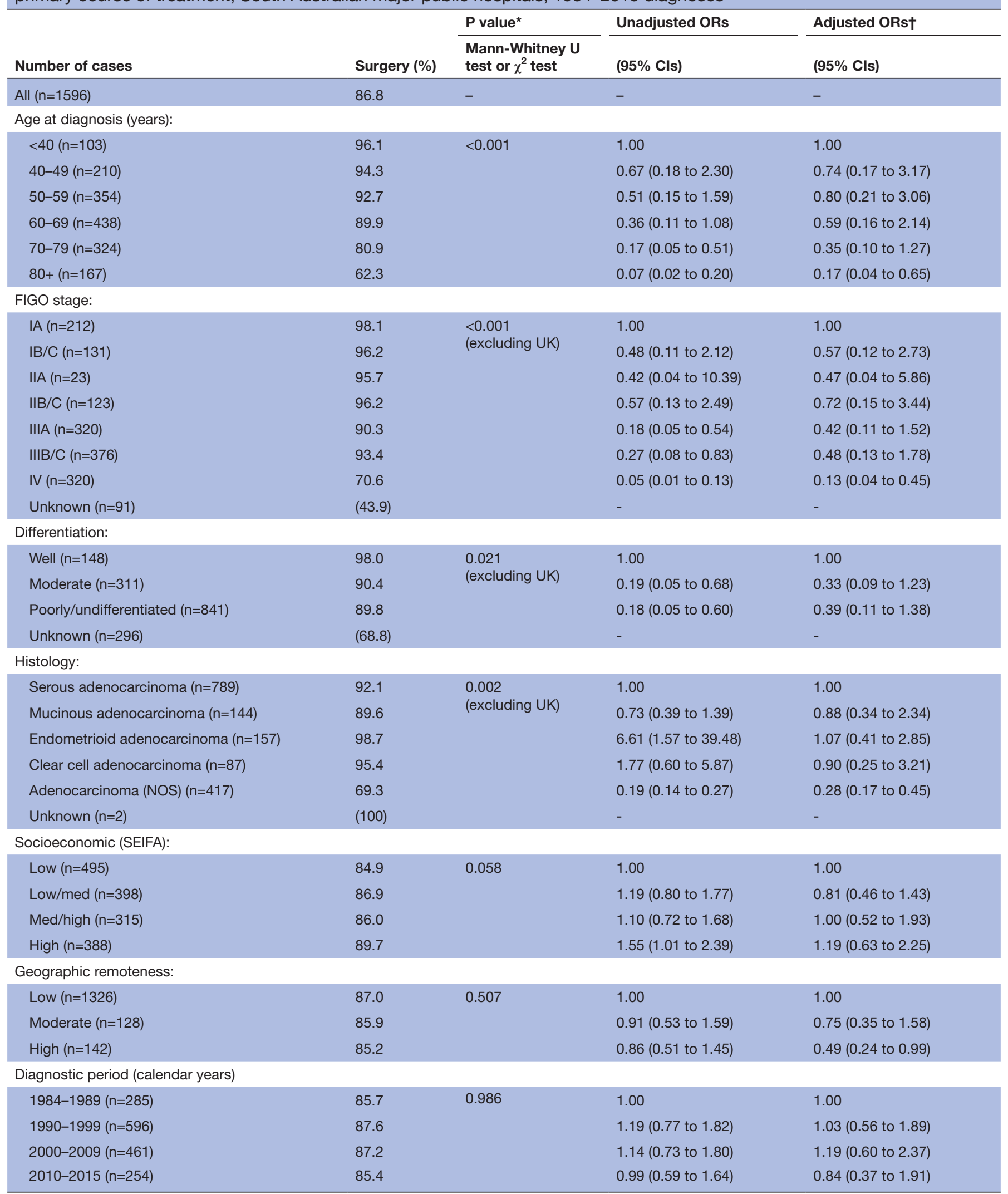

*Pearson's $\chi^{2}$ (likelihood ratio), Mann-Whitney $U$ test (see 'Methods' section).

†Derived from multiple logistic regression, adjusting for the other variables in the table.

FIGO, International Federation of Gynecology and Obstetrics; NOS, not otherwise specified; SEIFA, Socio-Economic Indexes for Areas; UK, Unknown. 
Table 3 Per cent cancers of the ovary, fallopian tube and peritoneum having radiotherapy (and ORs of radiotherapy) as part of the primary course of treatment; South Australian major public hospitals, 1984-2015 diagnoses

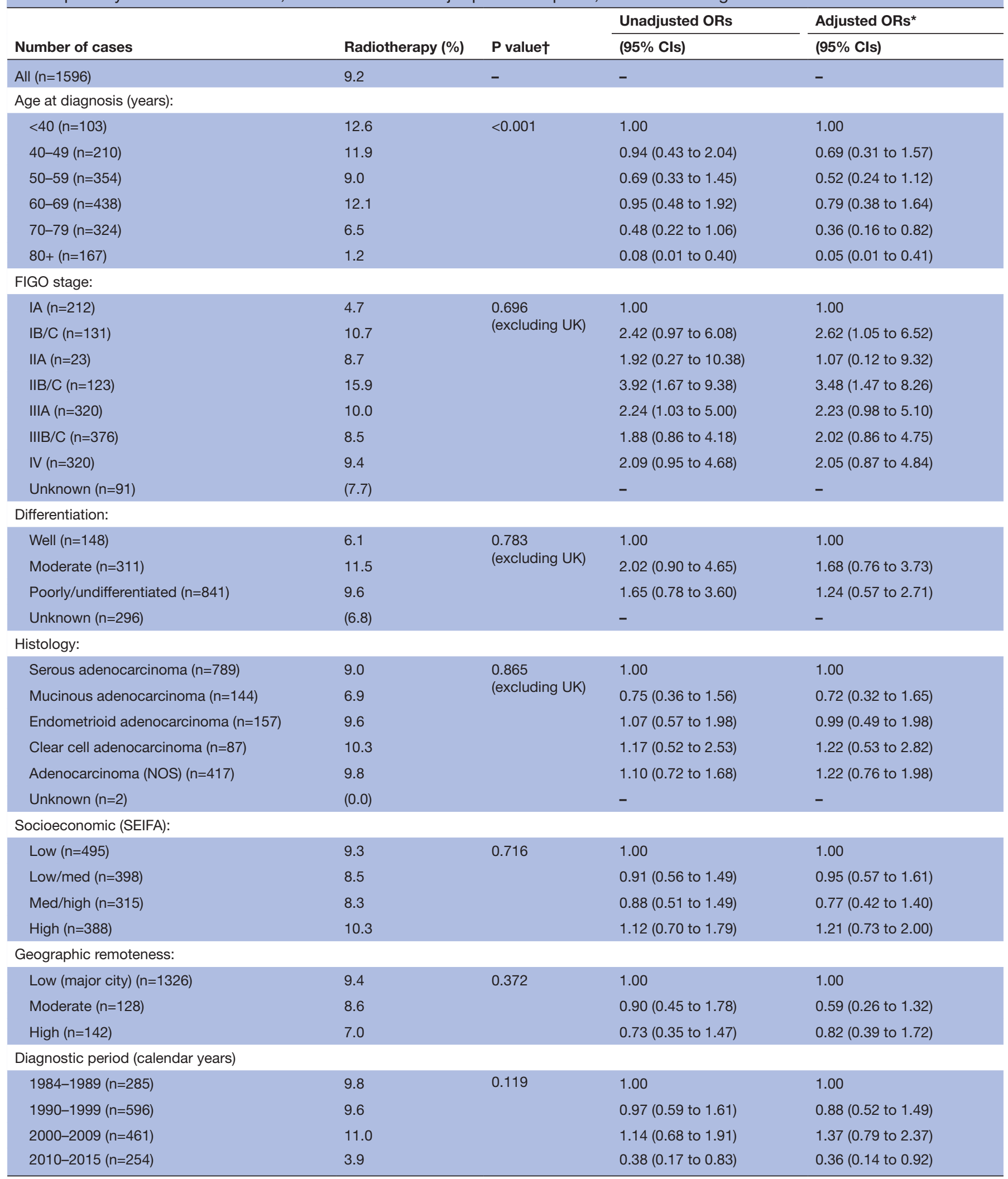

*Derived from multiple logistic regression, adjusting for the other variables in the table.

†Pearson's $\chi^{2}$, Mann-Whitney U test (see 'Methods' section).

FIGO, International Federation of Gynecology and Obstetrics; NOS, not otherwise specified; SEIFA, Socio-Economic Indexes for Area; UK, unknown. 
Table 4 Per cent cancers of the ovary and fallopian tube having systemic therapy (and ORs of systemic therapy) as part of the primary course of treatment; South Australian major public hospitals, 1984-2015 diagnoses

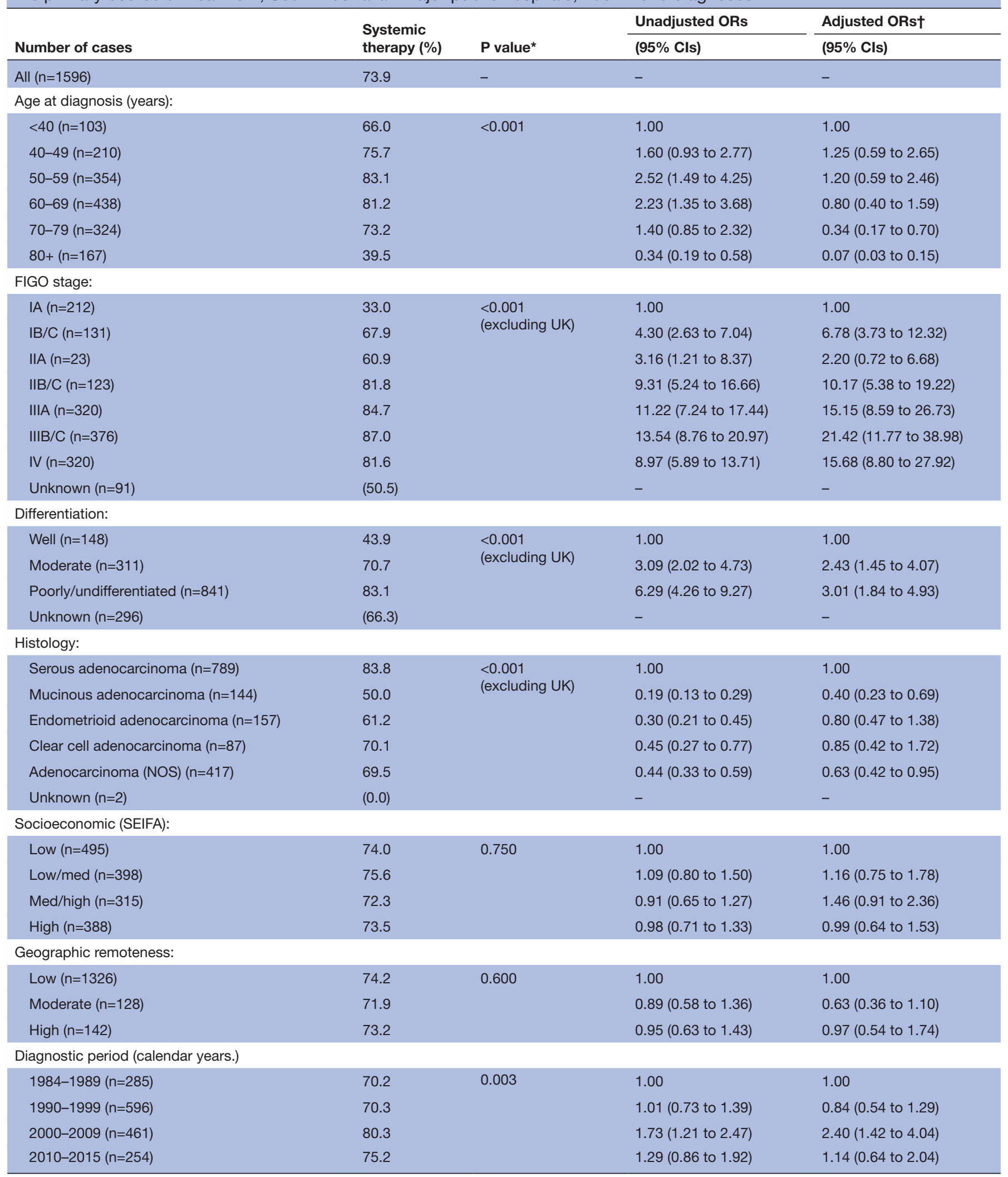

*Pearson's $\chi^{2}$, Mann-Whitney U test (see 'Methods' section).

†Derived from multiple logistic regression, adjusting for the other variables in the table.

FIGO, International Federation of Gynecology and Obstetrics; NOS, not otherwise specified; SEIFA, Socio-Economic Indexes for Area; UK, unknown. 
3.06). Differences were not found by geographic measures of remoteness or socioeconomic disadvantage (table 4).

\section{DISCUSSION}

Five-year cause-specific survival in this study was similar (44\% for 2010-2015) to the corresponding relative survival in 2009-2013 for Australia overall $(44 \%)^{3}$ and the USA (SEER: $47 \%$ for 2007-2013) ${ }^{7}$ However, comparison between Australian and USA data were for ovary only, and included all histology types. Corresponding ovary data from our study for 2010-2015 gave a 5-year survival of $50 \%$.

FIGO stage showed $65 \%$ to have metastatic spread in 2010-2015, equivalent to the $63 \%$ for both staged SEER cases for $2007-2013^{7}$ and New South Wales (NSW) cases for $2008-2012,{ }^{19}$ and greatly exceeding the corresponding $20 \%$ for all solid cancers combined for NSW. ${ }^{19}$ The challenge of finding ovarian cancers earlier appears not to have been met, despite extensive attempts through use of vaginal ultrasound, cancer antigen 125 and other methodologies. ${ }^{20}$

Consistent with upward trends in survival for Australia ${ }^{26}$ and the USA, ${ }^{7}$ an increase in survival was observed in this study, with adjusted HRs reducing to 0.75 (95\% CI 0.57 to 0.98) for 2010-2015 compared with the 1984-1989. While there was the indication of an increased proportion of patients receiving systemic therapies, the trend was inconsistent. It is possible that the increase in survival reflected the use of more aggressive debulking surgery and of carboplatin and taxane-related chemotherapies. ${ }^{8}$ Local clinicians also report an increased use of neoadjuvant chemotherapy followed by interval debulking surgery for patients with extensive disease, comorbidities or advanced age. ${ }^{21}$ While targeted therapies, such as bevacizumab, are likely to lead to improved outcomes, these were not in general use for ovarian cancer in Australia during the 1984-2015 study period.

Generally, differences were not found in survival and treatment practices by geographic remoteness and socioeconomic disadvantage, suggesting equity in service delivery. Surgery was an exception, where the odds of this treatment were lower in highly remote areas (up to $1300 \mathrm{~km}$ from specialised treatment centres), which likely reflects access issues.

Older patients had a much higher risk of death from these cancers, probably reflecting increasing frailty and comorbidity, and less intensive treatment (eg, a lower exposure to surgery). The issue of trading off therapeutically the most ideal cancer treatment against patient frailty is a complex issue where more trial and observational research evidence is needed.

Survival by histology type was consistent with previous reports. Recent Canadian data indicated higher survival for endometrioid than serous adenocarcinomas. ${ }^{22}$ Also, we found intermediary survival for mucinous lesions, as reported previously. ${ }^{23}$ While histology type was associated with type of clinical management, these associations may be largely indirect, reflecting correlations of histology type with bulky nodes or other clinical factors of relevance in treatment planning. ${ }^{24}$

The higher survival for endometrioid cancers was partly but not fully explained by stage and grade. This may relate to their biology, although this is not well-defined. Different causal factors and promoters also seem to be involved with these cancers, with oestrogenic factors playing a stronger role. ${ }^{25}$ Artificial factors are also possible, including changes in recording of histology type depending of pathologist specialisation.

The present data show treatment differences and trends consistent with clinical guidelines,${ }^{24}$ including a greater use of surgery for local and regional than disseminated cancers where opportunities for surgical removal would have been limited. Patients not receiving surgery tended to be older where higher levels of frailty and comorbidity would be expected.

By comparison, systemic therapies were used more frequently for more advanced cases. A trend was apparent for increased use of systemic therapy in 2000-2015 than for earlier diagnostic periods. Again, adjusted analyses indicated that where systemic therapy was not used, patients more often were aged $80+$ years where comorbidity would have been more prevalent and life expectancy shorter independently of the cancer. Radiotherapy was seldom used (applying to only 4\% of patients in 2010-2015), which is consistent with guideline recommendations. ${ }^{24}$

In this study, hospital-registry data enabled a greater depth of analysis and greater clinical insights than was possible with population-based registry data that generally exclude data on stage, other prognostic and treatment characteristics. Ideally, hospital-registry data would be extended to cover the entire population, as in Sweden. ${ }^{26}$ Increased interest exists in recording cancer stage in population-based registries and linking these data to inpatient and health insurance treatment data in Australia. ${ }^{27}$ The potential also exists to transfer other prognostic data electronically to registries from structured (synoptic) pathology reports. ${ }^{28}$ These population-based registry extensions are still at an early research and development phase and are yet to include ovarian cancers. Hospital-registry data will be valuable, where they exist, to validate these emerging population-based data, and to add value with additional clinical detail.

By comparison, population-based data will have greater geographic reach and include larger numbers of Aboriginal and ethnically diverse women, enabling greater statistical power. The data could be broadened to include comorbidity data to investigate effects on treatment and survival. ${ }^{28}$ Although the roles of population-based and hospital -registries would differ across populations, they have important complementary contributions to make.

This present study illustrates the added value of hospital-registry data for summarising prognostic and clinical management characteristics in local hospital environments and survival outcomes. While more detailed data have been published in special studies based on 
medical-record review, and have contributed significantly at a population level, ${ }^{29}{ }^{30}$ the present hospital-registry data are complementary in providing a longer view of trends in management and outcomes for specific hospital settings.

\section{CONCLUSIONS}

1. Results show the value adding of hospital-registry compared with population-basedregistry data for assessing local service delivery and outcomes.

2. The equivalent survival outcomes at study hospitals to Australia-wide and USA SEER survival, and the temporal gains observed after adjusting for stage and other patient characteristics, are reassuring.

3. Stage distributions are advanced and have not improved at study hospitals over time, underscoring the need for improved detection of preclinical markers and of early invasive disease.

4. Survival gains may reflect therapeutic benefits, potentially more extensive surgery and improved chemotherapy regimens. Increased debulking for local disease and systemic therapy for widespread disease align with guidelines and may explain these survival gains. Means of further supporting access to the latest treatments and guideline implementation may be indicated.

5. Generally, inequalities in outcomes and systemic therapy provision were not observed but the lower exposure to surgery of residents of remote rural areas (adjusted) and of the lowest that highest socioeconomic status (unadjusted), raises the question of service barriers. This requires further investigation and remedial action.

6. Older women have less surgical treatment and a lower survival. More research is needed to facilitate complex trade-off decisions between therapeutically ideal cancer care and raised levels of comorbidity and frailty.

7. Hospital-cancer registries complement population-based registries for evaluating local health-system activity and survival. They have an important contribution to make in cancer control.

\section{Author affiliations}

${ }^{1}$ Cancer Epidemiology and Population Health, University of South Australia, Adelaide, South Australia, Australia

${ }^{2}$ Private Consultant, Norwood, South Australia, Australia

${ }^{3}$ Royal Adelaide Hospital Cancer Centre, Adelaide, South Australia, Australia

${ }^{4}$ Flinders Medical Centre, Bedford Park, South Australia, Australia

${ }^{5}$ Lyell McEwin Hospital, Elizabeth Vale, South Australia, Australia

${ }^{6}$ Citi Centre Hindmarsh Square Adelaide, Adelaide, South Australia, Australia ${ }^{7}$ Population Health Research Group South Australian Health \& Medical Research Institute (SAHMRI), Adelaide, South Australia, Australia

${ }^{8}$ University of Adelaide, Adelaide, South Australia, Australia

${ }^{9}$ SA Clinical Cancer Registry, SA Health, Adelaide, South Australia, Australia

${ }^{10}$ Royal Adelaide Hospital, Adelaide, South Australia, Australia

\section{Acknowledgements Funding support is gratefully acknowledged.}

Contributors All authors contributed to the study conception, design and interpretation of the data. Data acquisition: KP, KF, DBT; quality control of data and algorithms: DR; data and statistical analysis: DR, KF; interpretation of historic data coding: MD; gynaecological oncology expertise: MD, MO; medical oncology expertise: SS-N, SP, JA, DK; health administration expertise: DK; population health expertise: CM; cancer registry science expertise: KP, DB-T; data management: KF; epidemiology expertise: DR, KF; manuscript preparation: DR, MO. All authors contributed to the editing and review of the manuscript and approved the version to be published and agreed to be accountable for the work.

Funding This study received funding support from Cancer Council SA (Beat Cancer Project), South Australian Health and Medical Research Institute, University of South Australia and the South Australian Department of Health.

Competing interests None declared.

Patient consent for publication Not required.

Ethics approval Research ethics approval for this study was provided by the South Australian Department for Health and Ageing Human Research Ethics Committee (reference number: LNR HREC/17/SAH/2).

Provenance and peer review Not commissioned; externally peer reviewed.

Data sharing statement Direct access to the data and analytical files is not permitted without the expressed permission of the approving human research ethics committees and data custodians. Researchers interested in collaboration should contact the corresponding author with their expression of interest.

Open access This is an open access article distributed in accordance with the Creative Commons Attribution Non Commercial (CC BY-NC 4.0) license, which permits others to distribute, remix, adapt, build upon this work non-commercially, and license their derivative works on different terms, provided the original work is properly cited, appropriate credit is given, any changes made indicated, and the use is non-commercial. See: http://creativecommons.org/licenses/by-nc/4.0/.

\section{REFERENCES}

1. Ferlay J, Soerjomataram I, Ervik M, et al. GLOBOCAN 2012 v1.0, Cancer Incidence and Mortality Worldwide: IARC CancerBase No. 11 [Internet]. Lyon, France: International Agency for Research on Cancer. 2013 http://globocan.iarc.fr (accessed 6 Apr 2017).

2 Australian Institute of Health and Welfare (AlHW). Australian Cancer Incidence and Mortality (ACIM) books. Canberra: AlHW, 2013. http:// www.aihw.gov.au/acim-books/ (Accessed 6 Apr 2017).

3. Australian Institute of Health and Welfare. Cancer in Australia 2017. Cancer Series no. 101. Cat. No. CAN 100. Canberra: AlHW, 2017.

4. Malvezzi M, Carioli G, Rodriguez T, et al. Global trends and predictions in ovarian cancer mortality. Ann Oncol 2016;27:2017-25.

5. Yang HP, Anderson WF, Rosenberg PS, et al. Ovarian cancer incidence trends in relation to changing patterns of menopausal hormone therapy use in the United States. J Clin Oncol 2013;31:2146-51.

6. Australian Institute of Health and Welfare and Cancer Australia. Gynaecological cancers in Australia: an overview. Cancer series no. 70. Cat. No. CAN 66. Canberra: AlHW, 2012.

7. Howlader N, Noone AM, Krapcho M, et al. SEER Cancer Statistics Review, 1975-2014, National Cancer Institute. Bethesda, MD. based on November 2016 SEER data submission, posted to the SEER web site, April 2017 https://seer.cancer.gov/csr/1975_2014/ (accessed 6 Apr 2017).

8. Wright JD, Chen L, Tergas Al, et al. Trends in relative survival for ovarian cancer from 1975 to 2011. Obstet Gynecol 2015;125:1345-52.

9. South Australian Cancer Registry. Epidemiology of cancer in South Australia. Incidence, Mortality and Survival 1977 to 1999. Issue 22, Cancer Series. Adelaide: South Australian Department of Health, 2000.

10. Government of South Australia. South Australia: Health Care Act 2008 - Part 7. Version: 5.6. Adelaide: South Australian Government, 2017.

11. Australian Institute of Health and Welfare, Cancer Australia \& Australasian Association of Cancer Registries. Cancer survival and prevalence in Australia: cancers diagnosed from 1982 to 2004. Cancer Series no. 42 Cat. No. CAN 38. Canberra AlHW, 2008.

12. Roder D, Karapetis CS, Wattchow D, et al. Colorectal cancer treatment and survival: the experience of major public hospitals in South Australia over three decades. Asian Pac J Cancer Prev 2015;16:2431-40.

13. von Elm E, Altman DG, Egger M, et al. The Strengthening the Reporting of Observational Studies in Epidemiology (STROBE) Statement. Epidemiology 2007;18:800-4.

14. Bonett A, Roder D, Esterman A. Cancer case-survival rates for South Australia: a comparison with US rates and a preliminary investigation of time trends. Med J Aust 1988;148:556-9. 
15. Armitage P, Berry G, Mathews JNS. Statistical methods in medical research. 2nd edn. Oxford, UK: Blackwell Scientific Publications, 1987.

16 StataCorp LP. Stata Statistical Software. Release13. College Station, TX: StataCorp LP, 2014.

17. South Australian Cancer Registry. Cancer in South Australia 2004 - with incidence projections to 2007. Adelaide: South Australian Department of Health, 2007.

18. Roulson J, Benbow EW, Hasleton PS. Discrepancies between clinical and autopsy diagnosis and the value of post mortem histology; a meta-analysis and review. Histopathology 2005;47:551-9.

19. Cancer Institute NSW. New South Wales Cancer Statistics. Sydney: Cancer Institute NSW. 2017 http://www.statistics.cancerinstitute.org. au/ (accessed 6 April 2017).

20. Buys SS, Partridge E, Black A, et al. Effect of screening on ovarian cancer mortality: the Prostate, Lung, Colorectal and Ovarian (PLCO) Cancer Screening Randomized Controlled Trial. JAMA 2011;305:2295-303.

21. Makar AP, Tropé CG, Tummers P, et al. Advanced ovarian cancer: primary or interval debulking? Five categories of patients in view of the results of randomized trials and tumor biology: primary debulking surgery and interval debulking surgery for advanced ovarian cancer. Oncologist 2016;21:745-54.

22. Bouchard-Fortier G, Panzarella T, Rosen B, et al. Endometrioid carcinoma of the ovary: outcomes compared to serous carcinoma after 10 years of follow-up. J Obstet Gynaecol Can 2017;39:34-41.
23. Tracey EA, Roder DM, Francis J, et al. Reasons for improved surviva from ovarian cancer in New South Wales, Australia, between 1980 and 2003: implications for cancer control. Int J Gynecol Cancer 2009;19:591-9.

24. Ledermann JA, Raja FA, Fotopoulou C, et al. Newly diagnosed and relapsed epithelial ovarian carcinoma: ESMO Clinical Practice Guidelines for diagnosis, treatment and follow-up. Ann Oncol 2013;24:vi24-32.

25. Mørch LS, Løkkegaard E, Andreasen AH, et al. Hormone therapy and different ovarian cancers: a national cohort study. Am J Epidemiol 2012;175:1234-42.

26. Leandersson P, Granåsen G, Borgfeldt C. Ovarian cancer surgery - a population-based registry study. Anticancer Res 2017;37:1837-45.

27. Cancer Australia. Improving Cancer Data Stage, Treatment and Recurrence (STAR). Sydney: Cancer Australia, 2017. https:// canceraustralia.gov.au/research-data/cancer-data/improving-cancerdata. (Accessed 6 Apr 2017).

28. Roder DM, Fong KM, Brown MP, et al. Realising opportunities for evidence-based cancer service delivery and research: linking cancer registry and administrative data in Australia. Eur J Cancer Care 2014;23:721-7.

29. Anuradha S, Webb PM, Blomfield P, et al. Survival of Australian women with invasive epithelial ovarian cancer: a population-based study. Med J Aust 2014;201:283-8.

30. Jordan S, Steer C, DeFazio A, et al. Patterns of chemotherapy treatment for women with invasive epithelial ovarian cancer - a population-based study. Gynecol Oncol 2013;129:310-7. 